\title{
Ultrasensitive IgG quantification using DNA nano-pyramids
}

\begin{abstract}
Liang Yuan ${ }^{1,3}$, Marcella Giovanni ${ }^{1,3}$, Jianping Xie ${ }^{1}$, Chunhai Fan ${ }^{2}$ and David Tai Leong ${ }^{1}$
In addition to being a repository of genetic information, DNA is a bio-polymer that can be formed into various nanostructures. This profound ability to engineer various moieties has expanded its role from data storage to a structural biomaterial for sensing applications. In this study, we anchored DNA nano-pyramids (DPs) to gold electrodes for the electrochemical sensing of immunoglobulin G (IgG), an important antibody produced in response to infection. The pyramidal DNA structure not only avoids entanglement with neighboring probes through the use of spatially separating pendant probes but also reduces the local overcrowding effect with the overall enhanced packing of targets. The results from electrochemical impedance spectroscopy measurements also show that DP layer has better conductivity, with the hollow structure further facilitating electron transfer and increasing the sensitivity of electrochemical detection. We are able to selectively detect IgG in the presence of other proteins in an analyte solution. The limit of detection was $2.8 \mathrm{pg} \mathrm{ml}^{-1}$. Our ferrocene-labeled sandwich immunoassay works at $37{ }^{\circ} \mathrm{C}$ under a neutral $\mathrm{pH}$ environment. It also produces stable and reproducible signals even after storage for 1 week at $4{ }^{\circ} \mathrm{C}$, further demonstrating the potential of this sensing system for clinical applications.
\end{abstract}

NPG Asia Materials (2014) 6, e112; doi:10.1038/am.2014.46; published online 11 July 2014

\section{INTRODUCTION}

Diagnostic tests are an integral part of any medical investigation. The current plethora of available diagnostic tests can help clinicians to establish or exclude a specific disease (especially at early onset), stage a given disease and even assess the suitability of different therapies. Certain central nervous system illnesses, such as bacterial and viral infections of the brain and inflammatory multiple sclerosis, require ultra-sensitive measurements of immunoglobulin G ( IgG) in blood and cerebrospinal fluid. ${ }^{1,2}$ IgGs are produced by differentiated B cells. These antibodies control infections by binding specifically to antigens on foreign microbes or parasites and inactivating them. In the event of an earlier infection, the sensitive detection of incremental amounts of IgG confers a great clinical advantage by allowing the option to administer antibiotics early in the infection process. However, it is often difficult to achieve a rapid and reliable assay for IgG, because they often present themselves at ultra-low levels in the early stages of an infection. Many efforts have been devoted to maximize the signal and realize an ultrasensitive diagnosis using various signalamplification strategies by utilizing enzymes, nanoparticles and polymerization..$^{3-7}$ A recent strategy for the ultrasensitive detection of IgG proteins using a dual-signal-amplification method consisting of polymers and tyramide-labeled quantum dots was reported and yielded a nearly 10 -fold increase compared with unamplified methods and could reach detection limits at the picogram level. ${ }^{8}$
It is well-established that DNA stores genetic information important for life. However, DNA can also be viewed as a bio-polymer or biomaterial, because it possesses some of the predictability of chain propagation and materialistic properties. ${ }^{9}$ Coupled with the classical Watsons-Crick base pairing rules, interesting and useful nanostructures can be engineered and tuned. ${ }^{10-15}$ Several examples of applications include biotemplates for nanofabrication of various materials, ${ }^{12,16}$ nanomachines for reversible structural conversion, ${ }^{17,18}$ platforms for biosensing, ${ }^{19,20}$ nanocages for drug delivery ${ }^{21,22}$ and tools for in vivo imaging and cell biology. ${ }^{23,24}$ Particularly, an elegant three-dimensional (3D) architecture was developed in recent years to build a well-defined DNA tetrahedral structure with struts composed entirely of double-stranded DNA. ${ }^{14}$ It was also found that the same DNA structure could be modified by tunable stoichiometry. ${ }^{15}$ The DNA tetrahedral structure can be considered as one of the most practical DNA nanostructures, and it has witnessed the speedy development of DNA tetrahedron for biosensing. ${ }^{25-30}$

One of the major challenges in biosensors that greatly reduces device sensitivity is the limited access of the target molecules to the immobilized binding moieties on the active sensor surface. Due to the unavoidable cross-masking of binding moieties, it was difficult to maintain stable, unhindered access to the target molecules (Figure 1). Earlier, we described a DNA nano-pyramid (DP) used as an anchoring scaffold for an electrochemical sensor system that

\footnotetext{
${ }^{1}$ Department of Chemical and Biomolecular Engineering, National University of Singapore, Singapore, Singapore and ${ }^{2}$ Division of Physical Biology, and Bioimaging Center, Shanghai Synchrotron Radiation Facility, Shanghai Institute of Applied Physics, Chinese Academy of Sciences, Shanghai, China

${ }^{3}$ These two authors contributed equally to this work.

Correspondence: Professor DT Leong, Department of Chemical and Biomolecular Engineering, National University of Singapore, 4 Engineering Drive 4, Singapore 117585, Singapore.

E-mail: cheltwd@nus.edu.sg
}

Received 27 November 2013; revised 7 April 2014; accepted 8 May 2014 
was able to detect as low as $1 \mathrm{pm}$ of DNA target. ${ }^{25}$ These DNA nanostructure-decorated surfaces are suitable for bioassay for several reasons. DP can be synthesized in a rapid and cost-effective manner through self-assembly of oligonucleotides at different temperatures and is compatible with existing bioassays. The nanostructure also stabilizes the targeting moieties and enables the spatial separation of one targeting moiety from its neighbors by a distance of at least $4 \mathrm{~nm},{ }^{25}$ maximizing access to antigen molecules by the targeting moiety and circumventing the need for surface passivation. ${ }^{26}$ The covalent bonds between the Au electrode and the thiolated DNA vertices allow a strong anchor that can withstand rigorous washing, which further reduces the background signals. The stiffness of the double helical structure comprising the struts of the DP also reduces the lateral flexing of the overall structure, which further adds to the stability.

In this study, we use DPs to detect protein molecules through conventional sandwich immunoreaction. Anti-IgG was covalently coupled with a free-standing carboxyl group at the top vertex of the pyramid, and ferrocenecarboxylic acid was used to generate electrochemical signals. Ferrocene-based redox active species and detection have received more attention for electrochemical biosensing owing to their unique catalytic properties, remarkable electrochemical responses and controllable and easy preparation. ${ }^{31-33}$ Using this system, we detected as low as $2.8 \mathrm{pg} \mathrm{ml}^{-1}$ of IgG and demonstrated a selective and stable sensing system after a week of storage. Our proof-of-concept study shows that this method has a potential to be developed into a biocompatible, sensitive and selective analytical method based on DPs for clinical diagnostics.

\section{MATERIALS AND METHODS}

\section{Reagents and materials}

Rabbit IgG protein (IgG) (antigen, Atg), goat anti-rabbit IgG antibodies (anti-IgG, $A b$ ) and bovine serum albumin were purchased from SigmaAldrich (St Louis, MO, USA). Fibronectin were obtained from Invitrogen (Carlsbad, CA, USA). N-hydroxysuccinimide (NHS), 1-ethyl-3-(3-dimethylaminopropyl) carbodiimide hydrochloride (EDC), tris(2-carboxyethyl) phosphine hydrochloride (TCEP), 11-mercaptoundecanoic acid (MUA), N-2hydroxyethylpiperazine- $N^{\prime}$-(2-ethanesulfonic acid) (HEPES) were also purchased from Sigma-Aldrich. Ferrocenecarboxylic acid (FeC-COOH) was obtained from Santa Cruz Biotechnology, Inc. (Dallas, TX, USA). TrisBorate-EDTA (TBE) buffer ( $89 \mathrm{~mm}$ Tris, $89 \mathrm{~mm}$ boric acid, $2 \mathrm{~mm}$ EDTA, $\mathrm{pH}$ 8.3) was obtained from Vivantis Inc. (Oceanside, CA, USA). Ultrapure water $(18 \mathrm{M} \Omega \cdot \mathrm{cm}$ resistivity, Milli-Q) was used throughout the study. Wash buffers consisted of phosphate-buffered saline $\left(0.1 \mathrm{M} \mathrm{Na}_{2} \mathrm{HPO}_{4}, 0.1 \mathrm{M} \mathrm{NaH} \mathrm{NO}_{4}\right.$ and $0.1 \mathrm{M} \mathrm{KCl}, \mathrm{pH} 7.4$; PBS-10) and its 10-fold diluted preparation (PBS-1).

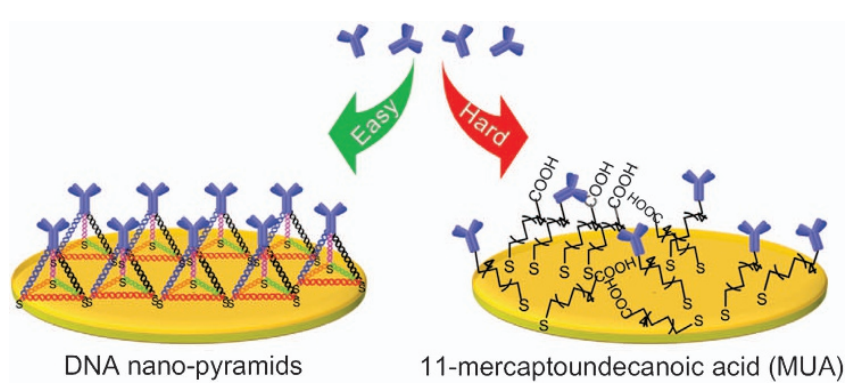

Figure 1 Schematic illustrating the ease of using Ab1 to immobilize on the electrode surface when using DPs versus a long-chain MUA molecule anchor. This ease of accessibility yields an overall improvement in the formation of the sandwich complex for the detection of the IgG antigen (not shown in this diagram).
All purified oligonucleotides were synthesized by AITbiotech Pte Ltd (Singapore) with the sequences of the oligomers shown in Supplementary Table S1 (Supplementary Information).

\section{Preparation of ferrocene-labeled anti-IgG conjugates (FeC-Ab2)}

Ferrocene-labeled anti-IgG (designated as $\mathrm{FeC}-\mathrm{Ab} 2$ ) was prepared through a typical carbodiimide coupling. ${ }^{34}$ Initially, FeC-COOH $(2 \mathrm{mg})$ was thoroughly dissolved in $700 \mu \mathrm{l}$ of HEPES buffer (50 mM, pH 7.3). A total of $10 \mathrm{mg}$ of NHS and $15 \mathrm{mg}$ of EDC were then added to the solution. The carboxyl groups on the $\mathrm{FeC}-\mathrm{COOH}$ in this mixture were then activated through continuous stirring for $2 \mathrm{~h}$ at room temperature. Afterwards, $300 \mu \mathrm{l}$ of $10 \mu \mathrm{g} \mathrm{ml}^{-1}$ anti-IgG (denoted Ab2) in PBS-1 ( $\mathrm{pH}$ 7.4) was added dropwise to the mixture, and continuously stirred for another $12 \mathrm{~h}$ at room temperature. After incubation, the conjugates were centrifuged for $10 \mathrm{~min}$ at 5000 r.p.m. to remove any precipitates and then spun purified in an Amicon Ultra centrifuge tube (MWCO-10K, $0.5 \mathrm{ml}$; Merck Millipore, Billerica, MA, USA). The FeC-Ab2 conjugate prepared in this manner was diluted in water to a final volume of $1 \mathrm{ml}$ and stored at $4{ }^{\circ} \mathrm{C}$.

\section{Synthesis of the DP}

The DPs were self-assembled by using methods described in previous reports. ${ }^{25,27}$ Four DNA strands (Pyra -A, Pyra -B, Pyra -C and Pyra -D) were diluted with TE buffer (10 mM Tris, $1 \mathrm{~mm}$ EDTA, pH 8.0) to a common final concentration of $50 \mu \mathrm{m}$. The obtained four oligonucleotides solutions were mixed in equimolar quantities in TM buffer $\left(20 \mathrm{~mm}\right.$ Tris, $50 \mathrm{mM} \mathrm{MgCl}_{2}$, pH 8.0), heated to $95^{\circ} \mathrm{C}$ for $2 \mathrm{~min}$ and then cooled to $4{ }^{\circ} \mathrm{C}$ over $30 \mathrm{~s}$ using a MJ Mini Personal Thermal Cycler (Bio-Rad Laboratories Ltd, Singapore). The product obtained was analyzed using non-denaturing polyacryamide gel electrophoresis $(12.5 \%)$ in TBE buffer at a constant voltage of $150 \mathrm{~V}$ at $4{ }^{\circ} \mathrm{C}$ for $2 \mathrm{~h}$. For one control experiment, the DP with no appended carboxyl group (f-DP) was also synthesized using Pyra-Free, Pyra-B, Pyra-C and Pyra-D using the same procedure.

Immobilization of DP nanostructures on the Au electrode surface The gold electrodes ( $\mathrm{Au}, 2 \mathrm{~mm}$ in diameter; $\mathrm{CH}$ Instruments, Austin, TX, USA) were polished according to the protocol used in a previous work. ${ }^{35} \mathrm{~A}$ volume of $2 \mu \mathrm{l}$ of each strand ( $50 \mu \mathrm{m}$ initial concentration in TE buffer) was mixed with $6 \mu \mathrm{l}$ of TCEP $(30 \mathrm{mM})$ and $36 \mu \mathrm{l}$ of TM buffer. The resulting mixture was heated to $95^{\circ} \mathrm{C}$ for $2 \mathrm{~min}$ and then cooled in ice bath in $0.5 \mathrm{~min}$. Next, $3 \mu$ of the as-prepared DP were added to the freshly cleaned Au electrode surface and incubated overnight at room temperature. The DP-modified electrode (Au-DP) was washed thoroughly with PBS-1 and dried with $\mathrm{N}_{2}$ gas before continuing with the following immunoreactions.

\section{Fabrication of the DP-based immunosensor}

The carboxylic group at the top vertex of DP was used to conjugate the antiIgG for sandwich immunoreactions. A volume of $3 \mu \mathrm{l}$ of freshly prepared $200 \mathrm{~mm}$ EDC and $100 \mathrm{~mm}$ in PBS-1 (pH 7.4) was pipetted onto the Au-DP surface and left to react for $15 \mathrm{~min}$. After washing with PBS-1, the activated $\mathrm{COOH}$-terminated electrode was followed by treatment with $3 \mu \mathrm{l}$ of anti-IgG solution $\left(10 \mu \mathrm{g} \mathrm{ml}^{-1}\right.$, denoted $\left.\mathrm{Ab} 1\right)$ for $2 \mathrm{~h}$. Consecutively, the electrode was incubated in $1 \%$ bovine serum albumin solution at $4{ }^{\circ} \mathrm{C}$ for $10 \mathrm{~min}$ to block nonspecific binding sites or excess active groups on the electrode surface. The capture antibody modified electrode (Au-DP-Ab1) was rinsed with PBS-1 and then incubated with various concentrations of the target Atg solution $(3 \mu \mathrm{l})$ at $37^{\circ} \mathrm{C}$ for 40 min to capture the antigen, designated as Au-DP-Ab1-Atg. After a thorough washing with PBS-1 to remove any unbound IgG, $3 \mu$ of ferroceneconjugated anti-IgG (FeC-Ab2) was carefully pipetted onto the electrode surface and incubated for another $40 \mathrm{~min}$ at $37^{\circ} \mathrm{C}$ to expose $\mathrm{Ab} 2$ to the immobilized antigen on the electrode surface. The electrode was extensively rinsed again and gently washed with PBS-1 to remove any adsorbed FeC-Ab2. Electrochemical measurements were made on the ferrocene-immobilized sensor (Au-DP-Ab1-Atg-(FeC-Ab2)). 
a

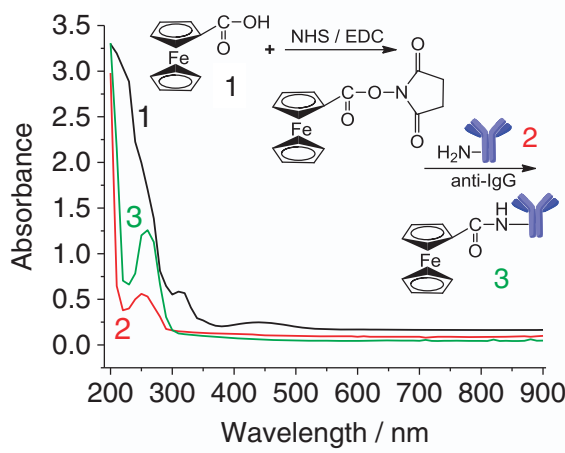

b

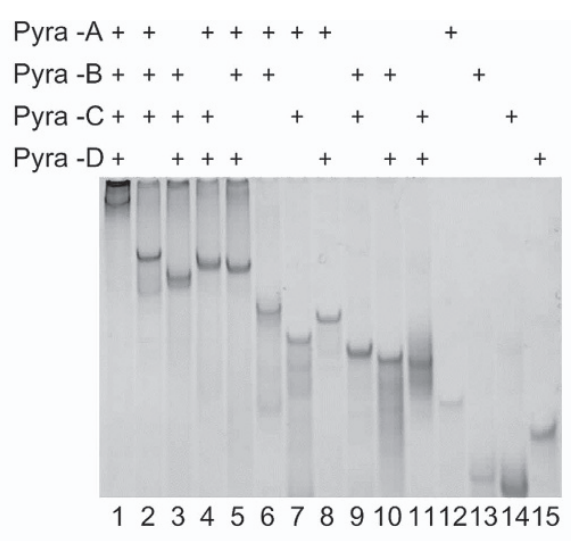

C

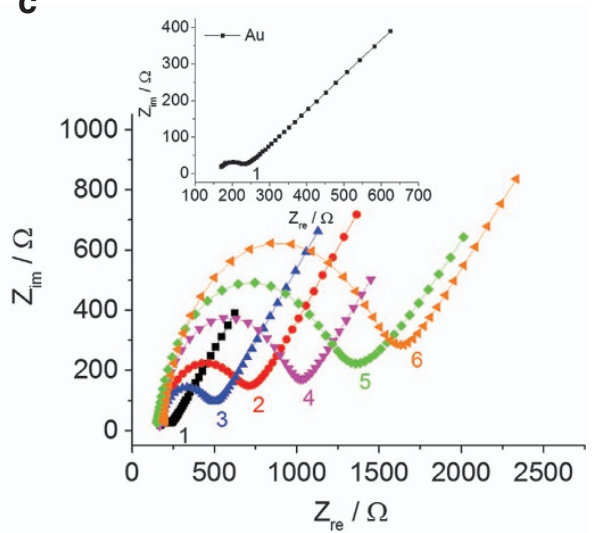

d

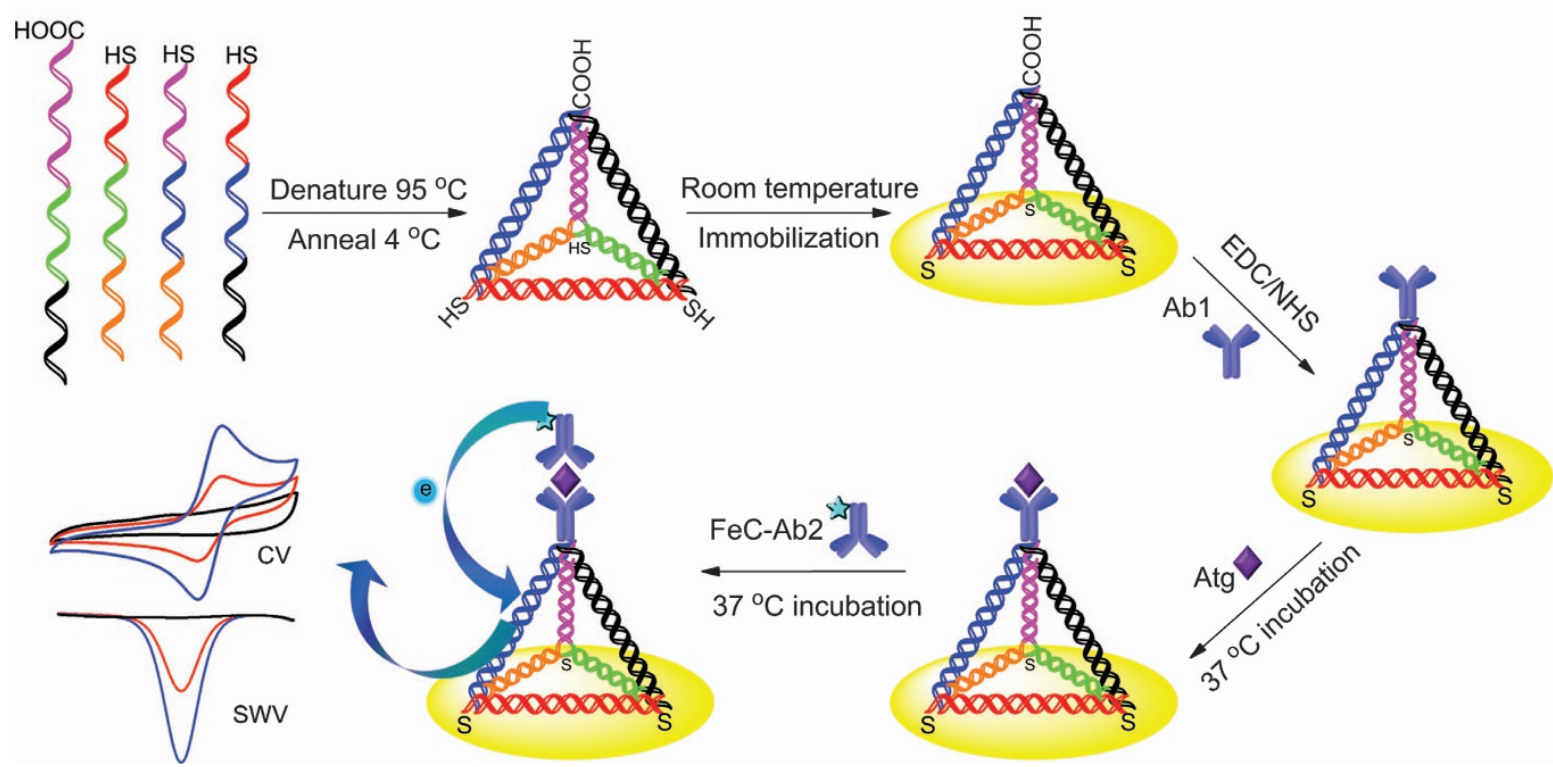

Figure 2 Formation and characterization of the DPs and associated electrochemical assemblies. (a) UV-vis absorption spectra of (1) ferrocenecarboxylic acid, (2) anti-lgG, and (3) FeC-anti-lgG. (b) Gel electrophoretic analysis of the formation of DNA tetrahedron. Lane 1 represents a COOH-terminated tetrahedron. The control experiment for single-stranded (ss-) DNA (lanes 12, 13, 14, 15) and other combinations lacking one strand (lanes 2, 3, 4, 5) or two strands (lanes $6,7,8,9,10,11)$. (c) Typical Nyquist plots of the same gold electrode in PBS-10 (pH 7.4) containing $5 \mathrm{~mm}\left[\mathrm{Fe}(\mathrm{CN})_{6}\right]^{3-1}$ $\left[\mathrm{Fe}(\mathrm{CN})_{6}\right]^{4-}$ and $0.1 \mathrm{~m} \mathrm{KCl}$ at different stages: (1) bare Au electrode, (2) the DP assembled Au-DP electrode, (3) the EDC/NHS activated Au-DP electrode, (4) the anti-IgG-immobilized Au-DP-Ab1 electrode, (5) the IgG antigen-immobilized Au-DP-Ab1-Atg electrode, and (6) ferrocene-labeled anti-IgG-immobilized Au-DP-Ab1-Atg-(FeC-Ab2) electrode. Inset: zoomed in Nyquist diagram of the bare Au electrode. (d) Schematic illustration of the sandwich immunoassay using the DP-modified Au electrode.

\section{Experimental measurements}

All electrochemical measurements were performed at $25^{\circ} \mathrm{C}$ using an Eco Chemie Autolab PGSTAT 30 electrochemical workstation (Metrohm B. V.). The data were analyzed with the provided General Purpose Electrochemical System software. A conventional three-electrode system, which involved a platinum wire counter electrode, an $\mathrm{Ag} / \mathrm{AgCl}(3 \mathrm{M} \mathrm{KCl})$ reference electrode and an Au-DP working electrode, was used throughout the experiments. All the potentials mentioned in this paper are referred to the $\mathrm{Ag} / \mathrm{AgCl}$ electrode.

Electrochemical impedance spectroscopy of the immunosensor fabrication was performed in PBS-10 (pH 7.4) containing $5 \mathrm{~mm}\left[\mathrm{Fe}(\mathrm{CN})_{6}\right]^{3-}$ $\left[\mathrm{Fe}(\mathrm{CN})_{6}\right]^{4-}$ and $0.1 \mathrm{M} \mathrm{KCl}$, with a biasing potential of $0.18 \mathrm{~V}$, an amplitude of $5 \mathrm{mV}$ and over a frequency range from $0.1 \mathrm{~Hz}$ to $100 \mathrm{kHz}$. Cyclic voltammetry was performed over a range from -0.2 to $0.6 \mathrm{~V}$ at a scan rate of $100 \mathrm{mVs}^{-1}$ in PBS-10 ( $\mathrm{pH}$ 7.4). Square wave voltammetric (SWV) measurements were also carried out in PBS-10 electrolyte solution ( $\mathrm{pH}$ 7.4) over a scan range from 0.6 to $0 \mathrm{~V}$ with a potential step of $4 \mathrm{mV}$, an amplitude of $25 \mathrm{mV}$ and at a frequency of $15 \mathrm{~Hz}$. All the measurements were performed in at least three independent experiments, and error bars correspond to the s.ds. from all of the measurements taken.
The UV-vis absorption spectra were recorded with an Epoch microplate spectrophotometer (BioTek Instruments, Singapore).

\section{RESULTS AND DISCUSSION}

Characteristics of FeC-anti-IgG conjugate (FeC-Ab2)

A schematic diagram of the synthesis of $\mathrm{FeC}-\mathrm{Ab} 2$ is shown in the inset of Figure 2a. The two coupling agents, EDC and NHS, were used to catalyze the formation of an amide bond between the amine group of the anti-IgG with the carboxyl group of the FeC-COOH. The UV-vis absorption spectra of the various components before and after the formation of FeC-anti-IgG are shown in Figure 2a. The pure FeC-COOH displayed two absorption peaks at 310 and $450 \mathrm{~nm}$ (curve 1, Figure 2a), whereas a characteristic peak at $250 \mathrm{~nm}$ was observed owing to anti-IgG antibody (curve 2, Figure 2a). However, after amide bond formation, the UV-vis spectra exhibited only one absorption peak at $265 \mathrm{~nm}$ (curve 3, Figure 2a), which can be attributed to the collapse of the $\mathrm{FeC}-\mathrm{COOH}(310 \mathrm{~nm})$ and anti-IgG $(250 \mathrm{~nm})$ peaks 
a

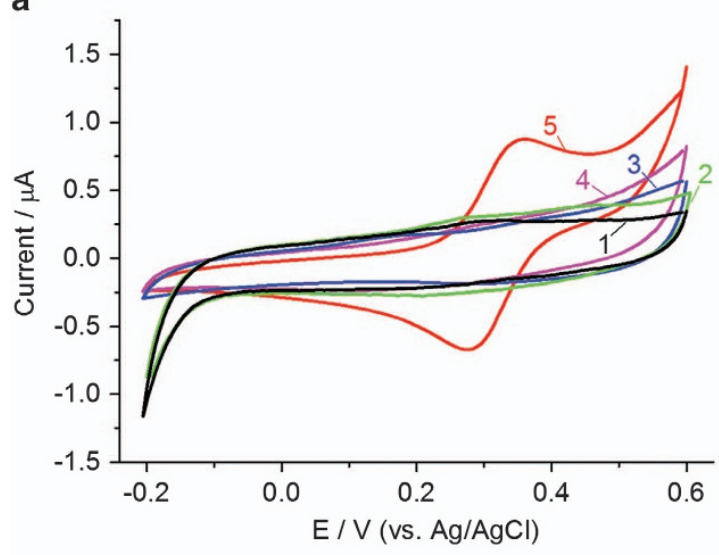

b

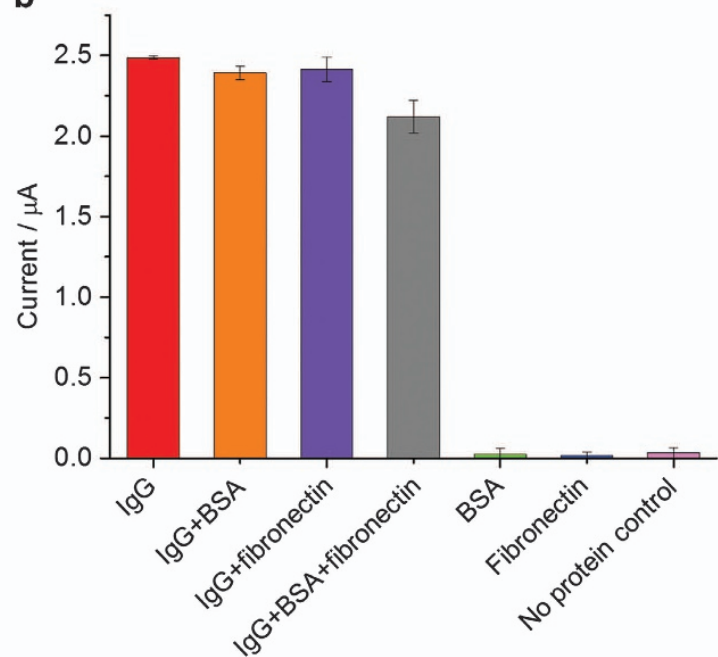

Figure 3 The DP-based sensor exhibited high specificity for IgG and low background signals even in the presence of non-specific proteins. (a) Cyclic voltammograms of the different surface-functionalized Au electrodes: (1) a bare Au electrode, (2) the DP assembled Au-DP electrode, (3) the Au-DP-Ab1 electrode, (4) the Au-DP-Ab1-Atg-Ab2 electrode, and (5) the ferrocene-labeled Au-DP-Ab1-Atg-(FeC-Ab2) electrode, at a scan rate of $100 \mathrm{mvs}^{-1}$ with PBS-10 supporting electrolyte $(\mathrm{pH}$ 7.4). (b) The SWV current response of the DP nanostructure-based immunosensing system after being exposed to different proteins in PBS-1 ( $\mathrm{pH}$ 7.4). Concentration of each protein is $1 \mathrm{ng} \mathrm{ml}^{-1}$.

into a single peak. This indicated that the $\mathrm{FeC}$ molecule was successfully immobilized on the anti-IgG (Ab2).

In addition, the binding of $\mathrm{FeC}$ to anti-IgG (Ab2) was also proven by X-ray photoelectron spectra (Supplementary Figure S1). The Fe spectrum of $\mathrm{FeC}$ exhibited an $\mathrm{Fe} 2 \mathrm{p}$ peak signal at a binding energy of $704.8 \mathrm{eV}$ (Supplementary Figure S1, curve a). A weaker N 1s peak appeared at $397.4 \mathrm{eV}$ because of the possible $\mathrm{N}-\mathrm{H}$ group from the solvent HEPES. As expected, the XPS spectrum of anti-IgG showed an obvious $\mathrm{N}$ 1s peak at $396.8 \mathrm{eV}$ and no discernible $\mathrm{Fe} 2 \mathrm{p}$ peak. However, after further coupling with $\mathrm{FeC}$, the XPS spectra of FeC-Ab2 conjugate showed two significant $\mathrm{N} 1 \mathrm{~s}$ peaks and one wider Fe $2 \mathrm{p}$ peak at the same binding energy as anti-IgG and $\mathrm{FeC}$, respectively. The emergence of a new $\mathrm{N}$ peak may be due to residual NHS. The presence of both two elements confirmed that the small $\mathrm{FeC}$ molecules had been successfully conjugated to the antibody. Subsequently, this conjugate would be used as an ultrasensitive signal tag for electrochemical detection.

\section{Characteristics of DPs}

DP can be considered to be one of the most simplistic 3D DNA structures. $^{25,26}$ DPs were constructed by annealing four DNA oligonucleotides strands. The resulting product was analyzed by using $12.5 \%$ polyacryamide gel electrophoresis (Figure 2b). As demonstrated in Figure 2d, sequences of the same colors were hybridized to form the six edges or struts of the pyramid. DPs (Figure 2b, lane 1) migrated more slowly than the various individual single-stranded DNA bands (Figure 2b, lanes 12-15) and other various combinations (that lacked either one or two individual strands groups-Figure 2b, lanes 2-11). Our results compared well to the relative sizes of the different species. The increased mass and spatial structure complexity of the DPs hindered the movements through the pores of the polyacrylamide gel, ${ }^{25}$ whereas the other structures with more compact or linear structures could pass through more easily. This confirmed the successful assembly of the complex DNA nanostructure. ${ }^{15,25}$

\section{Preparation of the DP-based immunosensor}

As shown in Figure 2d, three oligonucleotide strands were terminated with strategically placed thiol groups at three of the four vertices of the DP. The thiol group then covalently bonded the DP onto the Au electrode exactly at those bases. Before immobilization, TCEP was added into the hybridization solution to activate the thiolated DNA and reduce any disulfide linkages. The presence of TCEP did not interfere with the assembly, thus the obtained product could be used for subsequent experiments without any purification. Although it is expected that no reaction can be perfectly stoichiometric, any excess unassembled strands would not interfere with the coupling of assembled DP. ${ }^{35}$ The remaining non-thiolated vertex on the top of the DP allowed chemical coupling with different pendant probes to realize the biodetection (Figure 2d). In this study, we used a carboxyl group as a free-standing group at the top, and the non-thiolated vertex was covalently coupled with anti-IgG (Ab1). Through the immunobinding of $\mathrm{Ab} 2$ to Atg, this conventional sandwich immunoreaction allowed the electroactive tags (that is, $\mathrm{FeC}$ ) through Ab2 to be mobilized close to the electrode surface to generate significant electrochemical signals.

We used electrochemical impedance spectroscopy to prove each specific step in the sensor assembly process. As expected, the highfrequency region of the impedance plot shows a semicircular pattern. The depicted signals of the redox-probe $\left[\mathrm{Fe}(\mathrm{CN})_{6}\right]^{3-/ 4-}$ with the semicircle diameter represented the electron transfer resistance $\left(R_{\mathrm{et}}\right)$. Randles' equivalent circuit (inset in Supplementary Figure S2), was selected to reflect the real electrochemical process and determine the fitting impedance values that described our specific system. This resistance controlled the electron transfer kinetics of the redox-probe at the electrode interface. Therefore, the $R_{\text {et }}$ value $\left(R_{2}\right.$ in Supplementary Figure S2 inset) varies when different substances are immobilized on the electrode.

The Nyquist plots are shown in Figure 2c. The bare Au electrode, as expected, revealed a very small semicircular domain $\left(R_{\mathrm{et}}=56.4 \Omega\right.$, curve 1), implying a relatively low resistance to the redox probe. After the modification of the electrode with DPs, the resistance increased 
a
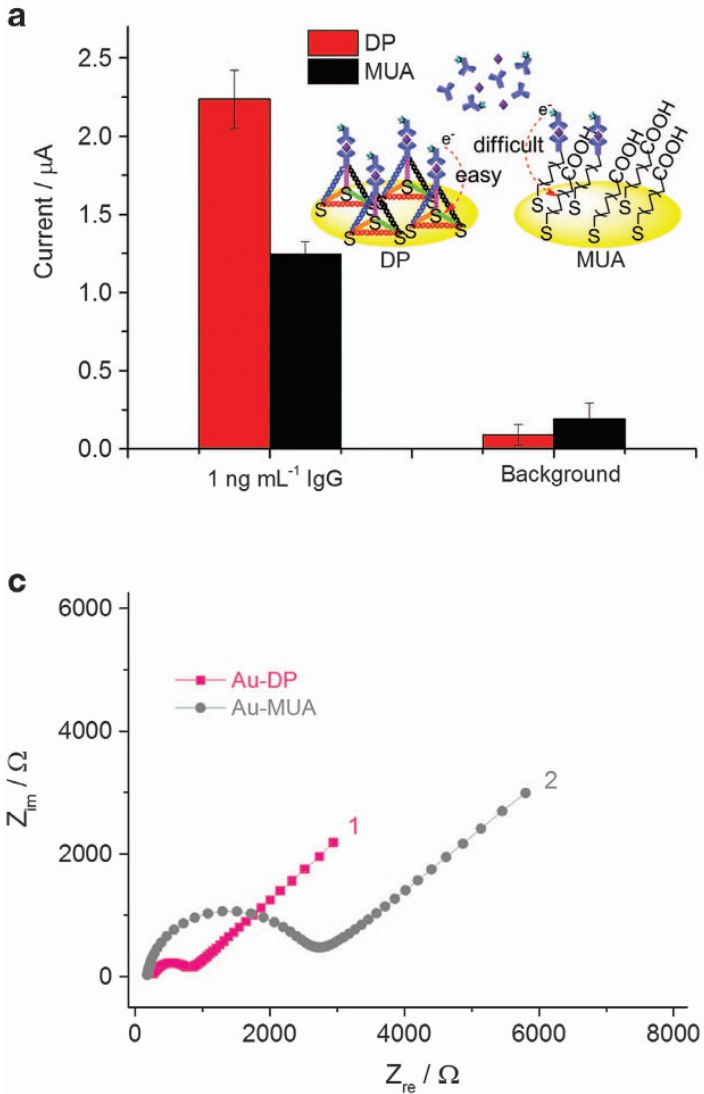

d

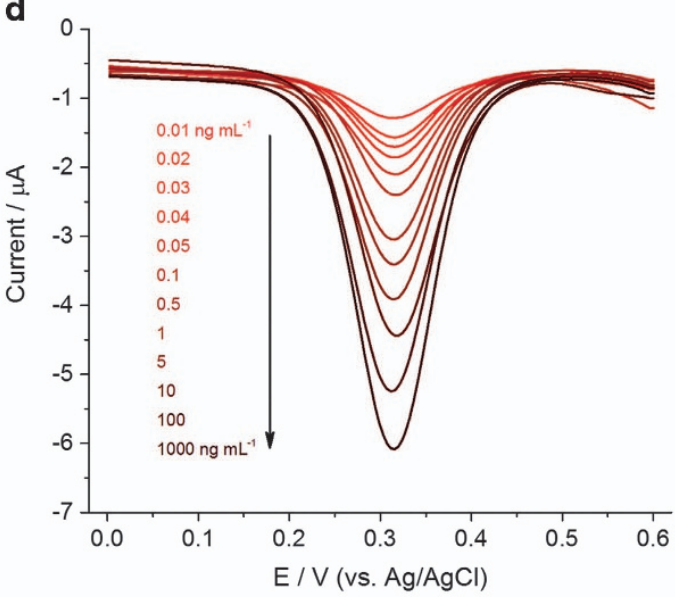

b
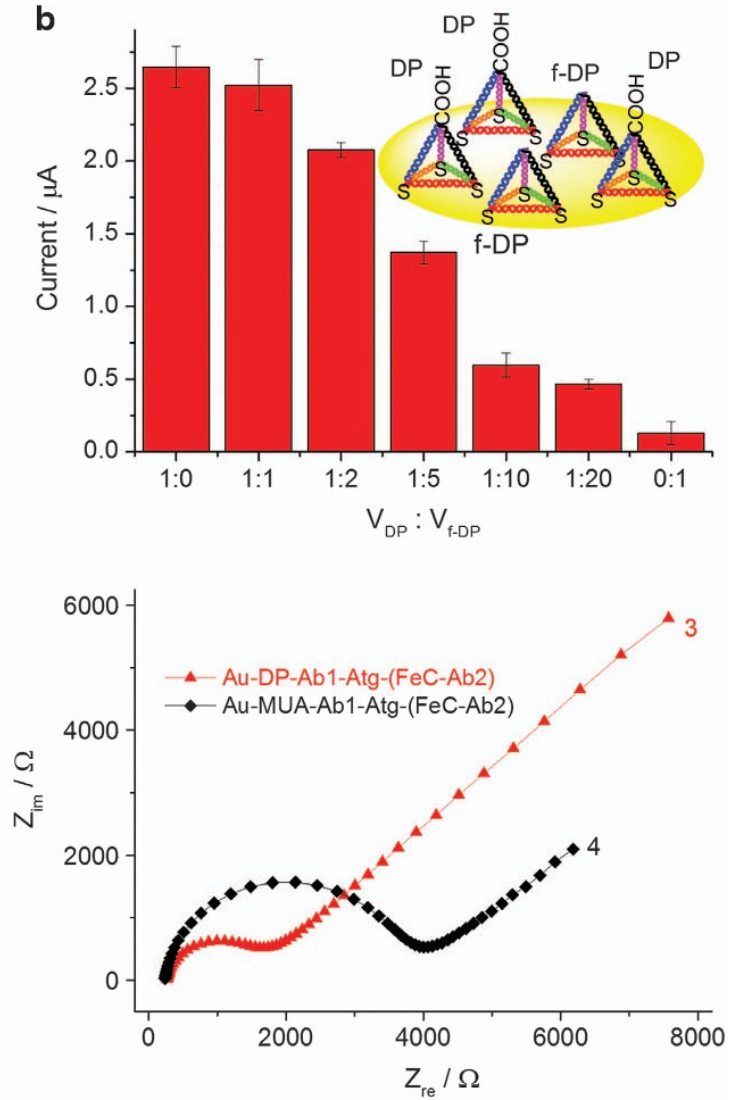

e

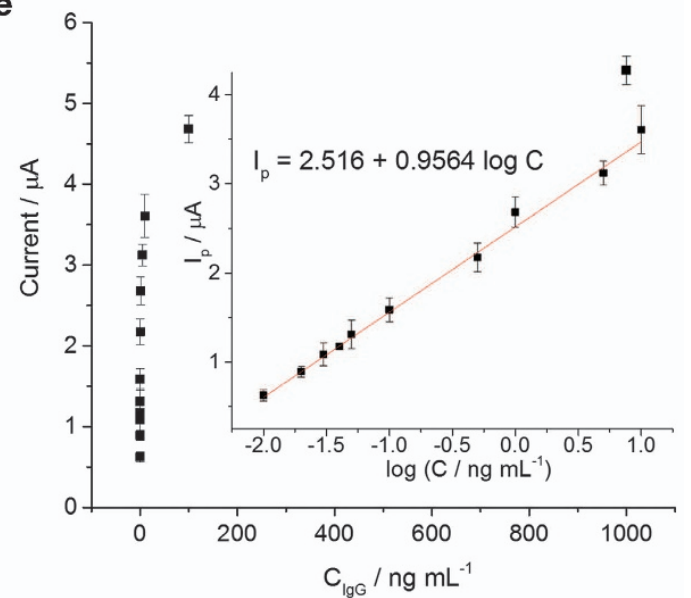

Figure 4 The presence of DPs increased the electrochemical signal magnitude and sensitivity for lgG detection. (a) Comparison of the DP-modified and thiolated MUA-modified electrodes in the IgG immunoassay. (b) The SWV response currents from the DP-based immunosensor at different ratios of DP and f-DP and constant DNA nanostructure total concentration. (c) Typical Nyquist plots for different modified Au electrodes in PBS-10 (pH 7.4 ) containing 5 mm $\left[\mathrm{Fe}(\mathrm{CN})_{6}\right]^{3-} /\left[\mathrm{Fe}(\mathrm{CN})_{6}\right]^{4-}$ and $0.1 \mathrm{~m} \mathrm{KCl}$ : (1) Au-DP, (2) Au-MUA, (3) Au-DP-Ab1-Atg-(FeC-Ab2) and (4) Au-MUA-Ab1-Atg-(FeC-Ab2). (d) SWV current curves of the DP nanostructure-based immunosensing system at different IgG concentrations of $0.01,0.02,0.03,0.04,0.05,0.1,0.5,1,5,10,100$ and $1000 \mathrm{ng} \mathrm{ml}^{-1}$. (e) Plot of the SWV peak current versus IgG in the incubation solution using this immunosensing system. Inset: the linear relationship between the change in the current response and the log of the IgG concentration.

appreciably $\left(R_{\mathrm{et}}=470.2 \Omega\right.$, curve 2$)$ compared with the bare $\mathrm{Au}$ electrode due to the relatively low poor conductivity of DP. This is further confirmed when the top vertex of DP was activated with EDC/ NHS solution, causing a decrease in the resistance $\left(R_{\mathrm{et}}=284.1 \Omega\right.$, curve 3) due to the neutralization of the DP's negative charge during the activation process. This allowed the conductive $\left[\mathrm{Fe}(\mathrm{CN})_{6}\right]^{3-14-}$ species to approach the activated Au-DP surface and accelerated the electron transfer between the redox moieties and the electrode. Subsequently, the activated Au-DP was incubated in Abl solution and the resistance increased again $\left(R_{\mathrm{et}}=708.4 \Omega\right.$, curve 4$)$ because of the insulating protein shell of Abl. Further coupling of the antigen and ferrocene-labeled antibody would generate a gradually thicker insulating layer and progressively increasing resistance values (detailed illustration in Supplementary Figure S2). These results confirmed that 
the fabrication of the electrode via sandwiched immunoreactions was achieved successfully. Interestingly, we also noted that the change in the resistance caused by the binding of FeC-Ab2 $\left(\Delta \mathrm{R}_{(6 \rightarrow 5)}=284.5 \Omega\right)$ was smaller than that of $\mathrm{Abl}\left(\Delta \mathrm{R}_{(4 \rightarrow 3)}=624.3 \Omega\right)$. Other than the slightly decreased number of binding sites on the antigen-immobilized electrode (Au-DP-Ab1-Atg) compared with Au-DP-(EDC/NHS) electrode, this is likely a consequence of using ferrocene as an electron mediator, which could favor the transfer of $\left[\mathrm{Fe}(\mathrm{CN})_{6}\right]^{3-/ 4-}$ species between the solution and electrode. To confirm this assumption, pure anti-IgG without FeC-COOH was directly captured on the Au-DPAb1-Atg electrode surface. The Nyquist diagrams of the two electrodes shows that with the help of ferrocene the resistance of the modified electrode clearly decreases (curve a, Supplementary Figure S3). However, this result also confirms the successful covalent coupling of ferrocene with the antibodies.

\section{Voltammetric characteristics of the DP-based immunosensor}

The key components in the biosensor included the pyramidal DNA nanostructure that anchored the recognition units on the electrode surface and an FeC-labeled antibody for signal generation. The electrochemical properties of $\mathrm{FeC}-\mathrm{Ab} 2$ in the electrolyte solution at DP-based electrode were investigated with voltammetric measurements. Figure $3 \mathrm{a}$ shows the cyclic voltammograms of the different modified electrodes in PBS-10. When the sandwich format reaction was completed, a pair of stable and well-defined redox peaks (curve 5, Figure 3a) could be observed at the Au-DP-Ab1-Atg-(FeC-Ab2)modified electrode, suggesting the efficient electrochemical activity of the FeC-anti-IgG bioconjugate labels. The DP structure did not interfere with electron communication between the ferrocene moieties and the underlying electrode. However, no peaks could be found on the bare Au electrode (curve 1, Figure 3a), DP assembled Au-DP electrode (curve 2, Figure 3a) or the capture antibody coupled Au-DP-Ablelectrode (curve 3, Figure 3a). To ensure the source of the signal, a control experiment was carried out using anti-IgG without any $\mathrm{FeC}$ label immobilized on the electrode. The absence of any discernible cyclic voltammetric peaks from the Au-DP-Ab1-AtgAb2 electrode (curve 4, Figure 3a) confirmed that the previously observed peaks were due to the presence of $\mathrm{FeC}$, as well as demonstrated a successful and complete immunoassay. By exploiting the unique structure and electronic properties of ferrocene, a specific target antigen can be sensitively detected. Additionally, the cyclic voltammograms from the prepared immunosensor varied at different scan rates (Supplementary Figure S4), which corresponded to the oxidation of the antibody-tethered ferrocene into the ferricinium cation. ${ }^{36}$ In addition, both the anodic and cathodic peak currents increased linearly with the square root of the scan rate within the range from 50 to $500 \mathrm{mV} \mathrm{s}^{-1}$ (inset of Supplementary Figure S4), which confirmed the diffusion-controlled nature of the redox species in this process. ${ }^{36}$ Despite earlier work that suggests that individual $\mathrm{FeC}$ assembled at the top vertex of a DP would yield poor current signals, we speculated the charge transfer mechanism in our current system may be that the ferrocene moieties on the Ab1-Atg-(FeC-Ab2) complex are flexible enough to physically impinge on the electrode surface and maintain a sufficiently high current signal. ${ }^{37}$

SWV was then used to characterize the performance of the immunosensor. Control experiments were conducted with the DP-based sensor in various protein solutions. When the Au-DPAbl electrode was incubated in $1 \mathrm{ng} \mathrm{ml}^{-1}$ of IgG antigen for $40 \mathrm{~min}$ followed by incubation in FeC-Ab2 suspension for another $40 \mathrm{~min}$, a remarkable SWV response peak of $2.48 \mu \mathrm{A}$ was obtained (curve a, Supplementary Figure S5). In contrast, after incubation in analyte solution without any protein but with subsequent $\mathrm{FeC}-\mathrm{Ab} 2$ incubation, no discernible SWV response was observed, and a peak current of only $0.034 \mu \mathrm{A}$ was observed (background signal current; Figure $3 \mathrm{~b}$ ). The ultralow signal might be caused by nonspecific but unavoidable adsorption. Next, to test the selectivity of the immunosensor, nontargeted bovine serum albumin and fibronectin at $1 \mathrm{ng} \mathrm{ml}^{-1}$ were each were added to the test systems. The results showed extremely low signals, similar to the background (Figure $3 \mathrm{~b}$ ). Simultaneously, when the immunosensor was challenged with different combinations of mixtures containing two or three proteins, the peak current variations resulting from the presence of the interfering proteins were $<10 \%$. This showed the high specificity of our DP-based immunosensor towards our antigen target.

To further substantiate the advantages of using a DP as the surfacecovering stabilized nanostructures, a negative control was tested using MUA directly anchored to the Au electrode via the same thio-Au binding chemistry. Similar to the DP-based sensor, the carboxylic group on MUA provided reacting sites capable of combining with the captured antibody (Ab1) and also with the IgG and ferrocene-labeled antibody to the electrode surface through similar sandwich immunoreactions. However, the immobilized FeC tags generated an electrochemical SWV response that was much lower than the response from DP-based immunosensor (Figure 4a). The poor performance of the MUA-based sensor was most likely a result of the disordered and unstable self-assembled MUA monolayer. We further hypothesized that this self-assembled MUA monolayer forms an incidental resistive film over the electrode surface, preventing the free flow of electrons to the surface (Figure $4 \mathrm{a}$ ). Other control experiments were carried out to investigate the differences between these two types of structures on the electrode surfaces. Figure $4 \mathrm{c}$ shows the impedances of the DPs and MUA modified Au electrodes with and without the presence of an immunoassay. As observed, the electron-transfer resistance of the MUA-anchored electrode was much higher than the DP-anchored electrode. Interestingly, the same result was obtained even after the sandwich assay was completed. This marked difference suggested that the Au-DP electrode had excellent electrochemical transduction capabilities by taking advantage of the hollow structure associated with the pyramidal DNA assembly. Because this open structure allows the shuttling of electrons between the electrode surface and the solution, the electro-active moieties could penetrate the assembled layer more easily. ${ }^{26}$ Additionally, the background signal increased significantly to approximately $0.19 \mu \mathrm{A}$, compared with $0.034 \mu \mathrm{A}$ from the DP-based immunosensor. The SWV current was $1.24 \mu \mathrm{A}$ for $1 \mathrm{ng} \mathrm{ml}^{-1}$ of $\mathrm{IgG}$ with a signal-to-noise ratio of only $\sim 6.5$, demonstrating the greater sensitivity of the pyramidal DNA sensor. We attributed this to the ability of DP to repel proteins and facilitate better orientation and accessibility for antibody coupling, as well as minimizing nonspecific adsorption. ${ }^{25}$ After the immunoassay, the MUA-layered electrode also displayed a higher impedance than the DP-modified electrode, which further supported the above viewpoint (Figure 4c).

Previous studies used diluents such as 11-mercapto-1-undecanol or mercaptohexanol to decrease the surface crowding and improve the overall sensitivity of electrochemical sensors. However, other strategies can also use free DP to achieve the same effect. ${ }^{28}$ We examined the influence of dilution on thiolated DP free of $-\mathrm{COOH}$ functional group (f-DP) (Supplementary Figure S6). Figure $4 \mathrm{~b}$ shows that the SWV responses from different surface densities of DP by tuning the ratio of DP/f-DP. No significant current enhancement from dilution could be observed, as expected. A possible reason is that the rigid and bulky DP results in low surface crowding, and anti-IgG is directly 
coupled to the $\mathrm{COOH}$-anchored surface without any extended DNA as a bridge to link the proteins after hybridization. Particularly, higher dilution ratios of DP/f-DP led to lower signals, resulting from the growing loss of surface-anchored targets and assay probes. Therefore, the DP platform could be directly used for protein detection without any additional surface micro-/nano-modification strategies. Given these advantages, it was confirmed that the DP-based immunosensor can provide a sensitive and specific approach for a reliable quantitative detection of target proteins.

Optimization of the analytical conditions of the immunosensor The $\mathrm{pH}$ of the working buffer influences the electrochemical response of the immunosensor. The formation of the immuno-complex on the surface of the electrode also depends on the incubation time and temperature. Therefore, all these parameters must be investigated to optimize the antigen-specific responses. To find an optimal reaction $\mathrm{pH}$, a series of electrolyte buffer solutions with $\mathrm{pH}$ ranges from 5.0 to 10.0 were prepared to perform immunosensory assays (Supplementary Figure S7A). The results showed that the SWV responses increased within the $\mathrm{pH}$ range from 5.0 to 7.0 but decreased as the $\mathrm{pH}$ increased further. It was found that the extreme $\mathrm{pH}$ conditions damage the immobilized proteins, especially in alkaline conditions. Thus, the optimal $\mathrm{pH}$ for this detection system is 7.4, which is a common $\mathrm{pH}$ level in the field of immunology.

Kinetically, it was expected that the antigens captured from the sample solution depended on the incubation time before reaching thermodynamic equilibrium. Supplementary Figure S7B illustrates the variation of the assay SWV signal with reaction time over a period up to $60 \mathrm{~min}$. The peak current intensities increased with the incubation time up to $40 \mathrm{~min}$ before reaching a plateau, indicating that the interactions between IgG and its cognate anti-IgG antibody reached saturation. As a result, a 40-min incubation time was adopted for this immunosensor.

Supplementary Figure S7C revealed the effect of test temperature on the biosensor performance over a range of $20-50^{\circ} \mathrm{C}$. The SWV current variation showed a gradual increase up to $35 \sim 37^{\circ} \mathrm{C}$ but then decreased sharply at higher temperatures. Because the peak analytical signal occurs at $37^{\circ} \mathrm{C}$, all further immunoassay experimentations were undertaken at $37^{\circ} \mathrm{C}$.

\section{The SWV detection of IgG}

The DP-based immunosensor was then challenged with different IgG solution concentrations. The corresponding electrochemical currents were recorded. Figure $4 \mathrm{~d}$ shows the SWV curves of the immunosensor after incubating with a series of IgG concentrations, covering a range of five orders of magnitude (from $10 \mathrm{pg} \mathrm{ml}^{-1}$ to $1000 \mathrm{ng} \mathrm{ml}^{-1}$ ), under the same optimum conditions. The peak currents increased monotonically with the IgG solution concentration, demonstrating the ability of our immunosensor to quantify the different concentrations of target IgG. Significantly, given the unavoidable non-specific adsorption, a background signal of $0.034 \mu \mathrm{A}$ was obtained from the negative control using PBS-1 solution in the absense of IgG. This did not affect the immunosensor for the ultrasensitive detection of IgG, because the current response generated from the detection of the lowest concentration of $10 \mathrm{pg} \mathrm{ml}^{-1}$ of IgG was higher than that in the blank PBS-1 sample. In other words, the signal from $10 \mathrm{pg} \mathrm{ml}^{-1}$ of $\operatorname{IgG}(0.6 \mu \mathrm{A})$ could easily be distinguished from the background noise. The calibration plots displayed a good linear relationship between the SWV peak currents and the logarithm of the analyte concentrations over a linear range from $10 \mathrm{pg} \mathrm{ml}^{-1}$ to $10 \mathrm{ng} \mathrm{ml}^{-1}$ of IgG. The linear curve was fitted to a regression equation $I_{\mathrm{p}} / \mu \mathrm{A}=2.516+0.9564 \log$ (c/ng ml ${ }^{-1}$ ) with a coefficient of determination of $R^{2}=0.996$, where $I_{\mathrm{p}}$ is the oxidation current and $\mathrm{c}$ is the $\operatorname{IgG}$ concentration in the incubation solution (inset of Figure 4e). The limit of detection of $2.8 \mathrm{pg} \mathrm{ml}^{-1}$, based on a signal-to-noise ratio of 3 from the present work, was lower than what was previously reported using immunosensors for the detection of IgG. ${ }^{7,38,39}$ Despite not having signalamplification chemistry incorporated into our system, we were able to obtain a limit of detection that is comparable to other systems that did use signal-amplification strategies. $8,39,40$

\section{Reproducibility and stability of the immunosensor}

This immunosensor for IgG is highly reproducible, as observed from the high intra-assay and inter-assay precision. The intra-assay precision was determined by assaying a common IgG concentration with five independently prepared immunosensors' replicate measurements, whereas the inter-assay precision was estimated by detecting the common $\operatorname{IgG}$ concentrations using five immunosensors on the same electrode. Relative s.ds. of $9.23 \%$ and $8.55 \%$ were obtained for the intra- and inter-assay, respectively, indicating good precision and reproducibility. We further investigated the storage stability of the Au-DP-Ab1-Atg-(FeC-Ab2) electrode after storing the modified electrode in PBS- 1 at $4{ }^{\circ} \mathrm{C}$ for 1 week. No significant SWV signal variation was observed when $1 \mathrm{ng} \mathrm{ml}^{-1}$ of IgG solution was detected using the stored and freshly modified electrodes, indicating that the as-prepared immunosensors have high storage stability and are promising for practical applications.

\section{CONCLUSIONS}

In this work, we successfully proposed an universal strategy for developing highly sensitive IgG immunoassays using novel 3D $\mathrm{DP}-\mathrm{FeC}$ sandwich immunoassays on Au electrodes. The vertices of these DP are chemically modified to present flexible moieties for stable surface anchoring and easy sensor pendent coupling. A ferrocene-labeled antibody setup was used to generate excellent electrochemical signals. Particularly, the pyramidal structure endowed the substrate surface with less overcrowding coverage and directly increased the analyte accessibility. The dilution of the surface density with additional reagents was also found to be insignificant. Both the higher rigidity of DP and its ability to repel proteins produced uniform and orientational surface assemblies, as well as lower background interference. In addition, the pyramid's hollow structure contributes to easy electron transfers between the electro-active species and the electrode surface, permitting this immunoassay system to achieve ultrasensitive detection of proteins. Considering the good biocompatibility and flexible chemical modification of the DPs, this proof-of-concept study of $\operatorname{IgG}$ detection indicates that this strategy offers a sensitive and promising method for future clinical diagnosis research and could be used to develop biosensors to push the detection limit boundaries of other biological targets.

\section{CONFLICT OF INTEREST}

The authors declare no conflict of interest.

\section{ACKNOWLEDGEMENTS}

We thank Professor En-Tang Kang and Professor Koon Gee Neoh for their technical help and useful discussions. We also acknowledge funding from Singapore Ministry of Education Tier 1 and 2 (R-279-000-350-112 and R-279-000-418-112) and NUS Cross Faculty grant (R-279-000-414-112). 
1 Luque, F. A. \& Jaffe, S. L. Cerebrospinal Fluid Analysis in Multiple Sclerosis. Int. Rev. Neurobiol. 79, 341-356 (2007)

2 Hjelholt, A. Carlsen, T. Deleuran, B. Jurik, A. G. Schiøttz-Christensen, B. Christiansen, G. \& Birkelund, S. Increased levels of IgG antibodies against human HSP60 in patients with spondyloarthritis. PLOS ONE 8, e56210 (2013).

3 Zhuo, Y., Yuan, P. X., Yuan, R., Chai, Y. Q. \& Hong, C. L. Bienzyme functionalized three-layer composite magnetic nanoparticles for electrochemical immunosensors. Biomaterials 30, 2284-2290 (2009).

4 Iijima, M., Matsuzaki, T., Yoshimoto, N., Niimi, T., Tanizawa, K. \& Kuroda, S. Fluorophore-labeled nanocapsules displaying IgG Fc-binding domains for the simultaneous detection of multiple antigens. Biomaterials 32, 9011-9020 (2011).

5 Yuan, X., Tay, Y. Q., Dou, X. Y., Luo, Z. T., Leong, D. T. \& Xie, J. P. Glutathione-protected silver nanoclusters as cysteine-selective fluorometric and colorimetric probe. Anal. Chem. 85, 1913-1919 (2013).

6 Zhang, Y. X., Islam, N., Carbonell, R. G. \& Rojas, Q. J. Specificity and regenerability of short peptide ligands supported on polymer layers for immunoglobulin $\mathrm{G}$ binding and detection. ACS Appl. Mater. Interfaces 5, 8030-8037 (2013).

7 Yuan, L., Wu, Y. F., Shi, H. Y. \& Liu, S. Q. Surface-initiated atom-transfer radical polymerization of 4-acetoxystyrene for immunosensing. Chem. Eur. J. 17, 976-983 (2011).

8 Yuan, L., Xu, L. L. \& Liu, S. Q. Integrated tyramide and polymerization-assisted signal amplification for a highly-sensitive immunoassay. Anal. Chem. 84, 10737-10744 (2012).

9 Andersen, E. S., Dong, M. D., Nielsen, M. M., Jahn, K., Subramani, R., Mamdouh, W., Golas, M. M., Sander, B., Stark, H., Oliveira, C. L. P., Pedersen, J. S., Birkedal, V., Besenbacher, F., Gothelf, K. V. \& Kjems, J. Self-assembly of a nanoscale DNA box with a controllable lid. Nature 459, 73-76 (2009).

10 Rothemund, P. W. Folding DNA to create nanoscale shapes and patterns. Nature 440 297-302 (2006)

11 Pei, H., Zuo, X. L., Pan, D., Shi, J. Y., Huang, Q. \& Fan, C. H. Scaffolded biosensors with designed DNA nanostructures. NPG Asia Mater. 5, e51 (2013).

12 Liu, D. S., Cheng, E. J. \& Yang, Z. Q. DNA-based switchable devices and materials. NPG Asia Mater. 3, 109-114 (2011).

13 Seeman, N. C. Feature DNA in a material world. Nature 421, 427-431 (2003).

14 Goodman, R. P., Schaap, I. A., Tardin, C. F., Erben, C. M., Berry, R. M., Schmidt, C. F. \& Turberfield, A. J. Rapid chiral assembly of rigid DNA building blocks for molecular nanofabrication. Science 310, 1661-1665 (2005).

15 Mitch, N., Schlapak, R., Kastner, M., Armitage, D., Chrzanowski, W., Riener, J., Hinterdorfer, P., Ebner, A. \& Howorka, S. A DNA nanostructure for the functiona assembly of chemical groups with tunable stoichiometry and defined nanoscale geometry. Angew. Chem. Int. Ed. 48, 525-527 (2009).

16 Zhang, C., Tian, C., Guo, F., Liu, Z., Jiang, W. \& Mao, C. D. DNA-directed threedimensional protein organization. Angew. Chem. Int. Ed. 51, 3382-3385 (2012).

17 Simmel, F. C. \& Dittmer, W. U. DNA nanodevices. Small 1, 284-289 (2005).

18 Modi, S., G, S. M., Goswami, D., Gupta, G. D., Mayor, S. \& Krishnan, Y. A DNA nanomachine that maps spatial and temporal $\mathrm{pH}$ changes inside living cells. Nat. Nanotechnol. 4, 325-330 (2009).

19 Rinker, S., Ke, Y. G., Liu, Y., Chhabra, R. \& Yan, H. Self-assembled DNA nanostructures for distance-dependent multivalent ligand-protein binding. Nat. Nanotechnol. 3, 418-422 (2008).

20 Fu, Y. M., Zeng, D. D., Chao, J., Jin, Y. Q., Zhang, Z., Liu, H. J., Li, D., Ma, H. W. Huang, Q., Gothelf, K. V. \& Fan, C. H. Single-step rapid assembly of DNA origam nanostructures for addressable nanoscale bioreactors. J. Am. Chem. Soc. 35 696-702 (2013).

21 Liu, X. W., Xu, Y., Yu, T., Clifford, C., Liu, Y., Yan, H. \& Chang, Y. A DNA nanostructure platform for directed assembly of synthetic vaccines. Nano Lett. 12, 4254-4259 (2012).

22 Kim, K. B., Kim, D. R., Lee, T., Yhee, J. Y., Kim, B. S., Kwon, I. C. \& Ahn, D. R. Drug delivery by a self-assembled DNA tetrahedron for overcoming drug resistance in breast cancer cells. Chem. Commun. 49, 2010-2012 (2012).

23 Kim, K. R., Lee, Y. D., Lee, T., Kim, B. S., Kim, S. \& Ahn, D. R. Sentinel lymph node imaging by a fluorescently labeled DNA tetrahedron. Biomaterials 34, 5226-5235 (2013).
24 Keum, J. W. \& Bermudez, H. Enhanced resistance of DNA nanostructures to enzymatic digestion. Chem. Commun. 45, 7036-7038 (2009).

25 Pei, H., Lu, N., Wen, Y. L., Song, S. P., Liu, Y., Yan, H. \& Fan, C. H. A DNA nanostructure-based biomolecular probe carrier platform for electrochemical biosensing. Adv. Mater. 22, 4754-4758 (2010).

26 Pei, H., Wan, Y., Li, J., Hu, H. Y., Su, Y., Huang, Q. \& Fan, C. H. Regenerable electrochemical immunological sensing at DNA nanostructure-decorated gold surfaces. Chem. Commun. 47, 6254-6256 (2011).

27 Wen, Y. L., Pei, H., Wan, Y., Su, Y., Huang, Q., Song, S. P. \& Fan, C. H. DNA nanostructure-decorated surfaces for enhanced aptamer-target binding and electrochemical cocaine sensors. Anal. Chem. 83, 7418-7423 (2011).

28 Wen, Y. L., Pei, H., Shen, Y., Xi, J. J., Lin, M. H., Lu, N., Shen, X. Z., Li, N. \& Fan, C. H. DNA nanostructure-based interfacial engineering for PCR-free ultrasensitive electrochemical analysis of microRNA. Sci. Rep. 2, 867 (2012).

29 Wang, Z. G., Xue, Q. W., Tian, W. Z., Wang, L. \& Jiang, W. Quantitative detection of single DNA molecules on DNA tetrahedron decorated substrates. Chem. Commun. $\mathbf{4 8}$ 9661-9663 (2012).

30 Zhang, M., Jiang, X. Q., Le, H. N., Wang, P. \& Ye, B. C. Dip-and-read method for labelfree renewable sensing enhanced using complex DNA structures. ACS Appl. Mater. Interfaces 5, 473-478 (2013).

31 Kang, D., White, R. J., Xia, F., Zuo, X. L., Vallée-Bélisle, A. \& Plaxco, K. W. DNA biomolecular-electronic encoder and decoder devices constructed by multiplex biosensors. NPG Asia Mater. 4, e1 (2012).

32 Yuan, L., Wei, W. \& Liu, S. Q. Label-free electrochemical immunosensors based on surface-initiated atom radical polymerization. Biosens. Bioelectron. 38, 79-85 (2012)

33 Wu, Z. S., Chen, C. R., Shen, G. L. \& Yu, R. Q. Reversible electronic nanoswitch based on DNA G-quadruplex conformation: a platform for single-step, reagentless potassium detection. Biomaterials 29, 2689-2696 (2008).

$34 \mathrm{Lim}$, T. K. \& Matsunaga, T. Construction of electrochemical flow immunoassay system using capillary columns and ferrocene conjugated immunoglobulin $\mathrm{G}$ for detection of human chorionic gonadotrophin. Biosens. Bioelectron. 16, 1063-1069 (2001).

35 Zhang, J., Song, S. P., Wang, L. H., Pan, D. \& Fan, C. H. A gold nanoparticle-based chronocoulometric DNA sensor for amplified detection of DNA. Nat. Protoc. 2 , 2888-2895 (2007)

36 Dou, Y. H., Haswell, S. J., Greenman, J. \& Wadhawan, J. Voltammetric immunoassay for the detection of protein biomarkers. Electroanalysis 24, 264-272 (2012).

37 Lu, N., Pei, H., Ge, Z. L., Simmons, C. R., Yan, H. \& Fan, C. H. Charge transport within a three-dimensional DNA nanostructure framework. J. Am. Chem. Soc. 134 13148-13151 (2012).

38 Zhang, S. B., Zheng, F., Wu, Z. S., Shen, G. L. \& Yu, R. Q. Highly sensitive electrochemical detection of immunospecies based on combination of $\mathrm{Fc}$ label and PPD film/gold nanoparticle amplification. Biosens. Bioelectron. 24, 129-135 (2008).

39 Qian, J., Dai, H. C., Pan, X. H. \& Liu, S. Q. Simultaneous detection of dual proteins using quantum dots coated silica nanoparticles as labels. Biosens. Bioelectron. 28, 314-319 (2011)

40 Wang, J., Han, H. Y., Jiang, X. C., Huang, L., Chen, L. N. \& Li, N. Quantum dot-based near-infrared electrochemiluminescent immunosensor with gold nanoparticle-graphene nanosheet hybrids and silica nanospheres double-assisted signal amplification. Anal. Chem. 84, 4893-4899 (2012).

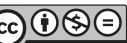

This work is licensed under a Creative Commons Attribution-NonCommercial-NoDerivs 3.0 Unported License. The images or other third party material in this article are included in the article's Creative Commons license, unless indicated otherwise in the credit line; if the material is not included under the Creative Commons license, users will need to obtain permission from the license holder to reproduce the material. To view a copy of this license, visit http://creativecommons.org/licenses/by-nc-nd/3.0/

Supplementary Information accompanies the paper on the NPG Asia Materials website (http://www.nature.com/am) 